\title{
Research Paper \\ Effect of a Lower Limb Restless Period on Expression of Mir-1 and Mir-206 Neural Muscle Genes in Endurance Training Rats
}

\author{
Mahboubeh Sheikhan ${ }^{1}$ (1) ${ }^{*}$ Mohammad Reza Kordi² ${ }^{2}$, Hamid Rajabi ${ }^{3}$ (1)
}

1. Department of Sports Physiology, Faculty of Physical Education and Sport Sciences, Kish Campus, University of Tehran, Kish, Iran. 2. Department of Sports Physiology, Faculty of Physical Education and Sport Sciences, University of Tehran, Tehran, Iran.

3. Department of Sport Physiology, Faculty of Physical Education and Sports Sciences, Kharazmi University, Tehran, Iran.

\begin{tabular}{|c|c|}
\hline $\begin{array}{l}\text { Use your device toscan } \\
\text { and read the article online }\end{array}$ & Citation: Sheikhan M, Kordi MR, Rajabi H. [Effect of a Lower Limb Restless Period on Expression of Mir-1 and Mir-206 \\
\hline 回幽回 & $\begin{array}{l}\text { Neural Muscle Genes in Endurance Training Rats (Persian)]. Journal of Arak University of Medical Sciences (JAMS). 2020; } \\
\text { 23(4): 570-579. https://doi.org/10.32598/JAMS.23.4.5947.1 }\end{array}$ \\
\hline 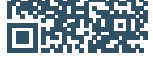 & doi) https://doi.org/10.32598/JAMS.23.4.5947.1 \\
\hline
\end{tabular}

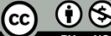

Article Info:

Received: 26 Jul 2019

Accepted: 10 Dec 2019

Available Online: 01 Oct 2020

Key words:

Muscular atrophy, Mi-

croRNAs, miR-1,

$\operatorname{miR}-206$

\section{AB STRACT}

Background and Aim Several microRNAs are involved in regulating muscle mass, which plays an essential role in hypertrophy and atrophy of skeletal muscle, The present study examined the expression of some genes as regulators of muscular atrophy following a period of inertia in rats.

Methods \& Materials For this purpose, 18 male Sprague-Dawley rats were divided into three groups (Control, Exercise+inactivity, and Inactivity). The exercise+inactivity group run on the treadmill for 18 weeks and five times per week. The hindlimb of the animal was immobilized for seven days with the casting method. Soleus muscle was extracted and the expression of the genes was measured by the RT-PCR method. Univariate ANOVA and Tukey post hoc test was used to determine the differences $(\alpha=0.05)$.

Ethical Considerations The Ethics Committee of the Tehran University of Medical Sciences Research approved this study (Code: IR.SUMS.REC.1396.S 463).

Results Results showed that immobilization in both Exercise+ inactivity and inactivity groups, compare to the control group, increased expression of miR-1 genes $(P<0.10), F O X O 3_{a}(P<0.001)$ and decreased expression of miR-206 ( $\mathrm{P}<0.007)$ and IGF-1 $(\mathrm{P}<0.001)$. This difference was statistically significant.

Conclusion According to the results of this study, it can be said that changes in the expression of RNAs by chromatography cause changes in the expression of muscle regulating genes, and although endurance exercises have protective effects, they cannot prevent these changes.

\section{Extended Abstract}

\section{Introduction}

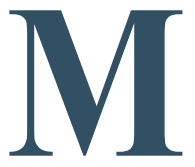

uscle atrophy is caused by the aging, inactive lifestyle, suspension, and a variety of pathological conditions [1]. Exercise can cause hypertrophy by inhibiting atrophic factors such as FOXO by developing the AKT pathway [3]. FOXO is inhibited by AKT and PGC-
$1 \alpha$ and decreased expression of PGC-1 $\alpha$ has also been observed in inactivity [4]. Little information is known about the role of miRNAs and their effects in the sedentary period after a training period [11]. $m i R-1$ and $m i R-206$ because they play an important role in increasing and inhibiting and on the other hand miR-1 is directly regulated by $\mathrm{FOXO3}$ and inhibits $I G F-1$ and in contrast $m i R-206$ through MyoD degradation was able to reduce $\mathrm{FOXO}_{a}$ The result is a lack of reduction in $I G F-1$ and ultimately maintenance of protein synthesis in inactivity [13]. This study aimed to

\section{* Corresponding Author:}

Mohammad Reza Kordi, PhD.

Address: Department of Sports Physiology, Faculty of Physical Education and Sport Sciences, University of Tehran, Tehran, Iran.

Tel: +98 (912) 3300076

E-mail: mrkordi@ut.ac.ir 
Table 1. Mean \pm SD of weight and food intake of groups and results of statistical test of analysis of variance

\begin{tabular}{|c|c|c|c|c|}
\hline \multirow{2}{*}{ Index } & \multicolumn{3}{|c|}{ Mean $\pm S D$} & \multirow{2}{*}{ One-way Analysis of Variance } \\
\hline & Control & Exercise + Inactivity & Inactivity & \\
\hline Body weight (g) & $363.6 \pm 14$ & $341.4 \pm 14$ & $337.6 \pm 08$ & ----- \\
\hline Soleus muscle (mg) & $220.6 \pm 11$ & $231.2 \pm 9$ & $189.2 \pm 12+$ & $\begin{array}{l}F=30.614 \\
\text { Sig:0.002 }\end{array}$ \\
\hline Soleus muscle / body weight (mg / kg) & $638.8 \pm 34$ & $690.8 \pm 35$ & $500.2 \pm 35+$ & $\begin{array}{c}F=162.050 \\
\text { Sig:0.016 }\end{array}$ \\
\hline Food intake (g / daily) & $23.8 \pm 3$ & $30.6 \pm 3$ & $14.4 \pm 5+$ & $\begin{array}{c}F=151.964 \\
\text { Sig:0.001 }\end{array}$ \\
\hline
\end{tabular}

$\dagger$ Significant changes compared to the trained group;

$\ddagger$ Significant changes compared to the sedentary group.

investigate the expression of $m i R-1, m i R-206, I G F-1$, and FoxO3 ${ }_{a}$ genes following a short period of inactivity in trained and untrained rats.

\section{Materials and Methods}

The present study was fundamental and experimental. A total of 18 male Sprague-Dawley rats at the age of eight weeks with an average weight of $200 \pm 20 \mathrm{~g}$ were randomly divided into control groups $(\mathrm{n}=6)$ and endurance training groups $(n=12)$. The rats in the endurance training group performed endurance training for 6 weeks and five days a week. Training started at 17.5 meters per minute for $15 \mathrm{~min}$ utes and reached 30 meters per minute for 60 minutes. The control group experienced walking on a treadmill at a speed of five meters per minute for 15 minutes [15]. The rats were anesthetized 48 hours after the last training session.
Both legs were then fixed at the hip, knee (in extension), and ankle (in plantar flexion) [16] for seven days. Immobilization of the lower extremities and the ability to freely consume water and food was confirmed by observation. In the end, all rats were anesthetized and soleus muscle was extracted and the expression of the desired genes was measured by the RT-PCR method and the ratio of the target gene to reference gene was calculated using CT-2 formula. To determine the differences, the one-way ANOVA method and Tukey post hoc test were used at a significant level $(\alpha \geq 0.05)$.

\section{Results}

Soleus muscle weight The ratio of horseshoe muscle weight to body weight and food intake of the exercise group compared to the sedentary-exercise + sedentary group were statistically significant (Table 1).

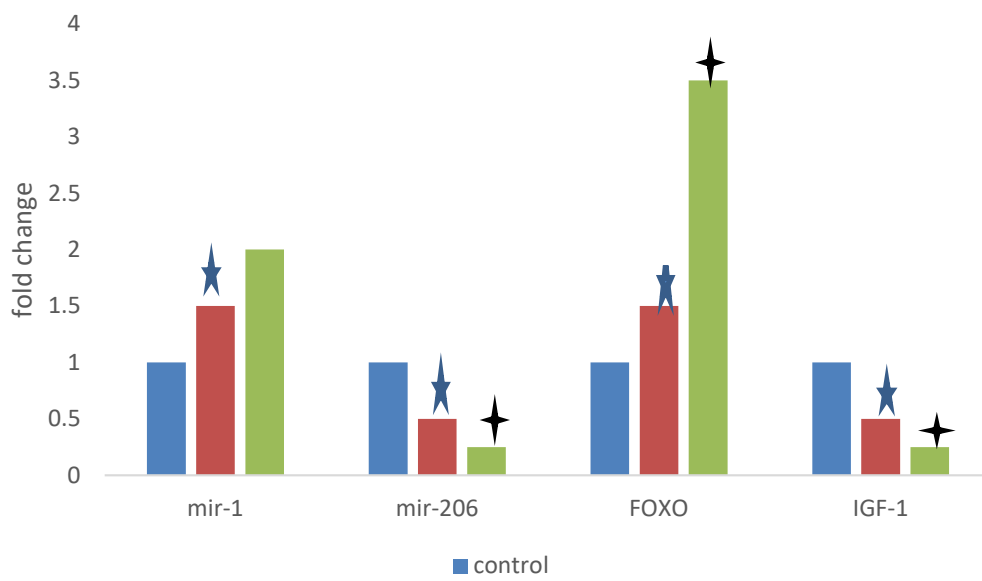

Figure 1. Changes in gene expression levels of groups and results of one-way analysis of variance with significant level $(\alpha \geq 0.05)$

$\star$ The sign indicates significant changes in the exercise+sedentary group compared to the control group;

+ The sign indicates significant changes in the sedentary group compared to the exercise+sedentary group. 
Increased expression of miR-1 ( $\mathrm{P}=0.012)$, FOXO3 $(\mathrm{P}=0.001)$ genes and decreased expression of miR-206 $(\mathrm{P}=0.007)$ and $I G F-1(\mathrm{P}=0.003)$ in the exercise+inactivity group and the inactivity group compared to the control group, these differences were statistically significant. Also, the in-group results showed a non-significant increase in $m i R-1$ gene expression $(\mathrm{P}=0.068)$ in the inactive group compared to the exercise+inactivity group, but an increase in FOXO3 ${ }_{a}(\mathrm{P}=0.004)$ in the inactive group compared to the exercise + group. Inactivity was significant. Also, the inactive group showed a significant decrease in the expression of miR-206 $(\mathrm{P}=0.030)$ and $I G F-1(\mathrm{P}=0.002)$ genes compared to the exercise + inactivity group (Figure 1).

\section{Discussion and Conclusion}

The most important findings of this study were the increase in the expression of atrophy stimulus genes ( $m i R-1$ and $\mathrm{FOXO}_{a}$ ) and the decrease in the expression of muscle hypertrophy stimulus genes (miR-206 and IGF-l) in the exercise+inactivity group and the inactivity group compared to the control group, which confirms the principle of training reversibility. Endurance exercise activities with increased aerobic performance and development of antioxidant capacity create favorable conditions for hypertrophy.

By decreasing reactive oxygen species and increasing PGC$1 \alpha$ expression, the $\mathrm{FOXO}_{a}$-associated atrophy signal pathways associated with $m i R-l$ are reduced. Exercise appears to increase $I G F-1$, which is associated with $m i R-206$. With inactivity, the first thing that happens in the muscles is a decrease in aerobic power, which has been shown to decrease PGC-1 $\alpha$ expression. This reduction reduces the intracellular content of muscle, especially intracellular water, which in turn reduces antioxidant defenses, and ultimately allows atrophic pathways to grow due to the absence of hypertrophic agents.

Despite the protective effects of exercise activities against atrophy due to inactivity, the effect of complete inactivity will be greater due to high levels of fitness. This process is achieved by reducing the stimulants of hypertrophy and increasing the inhibitory factors by reducing the antioxidant defense and will play a very important role in the development of growth hormones. In addition, very important cellular markers called $m i R-206$ are reduced, which causes the atrophy process to continue. In spite of the importance of preventing this regressive process and reducing ideal bodily function, there is still no clear way to deal with this inactive condition and the need for further studies and training (nutrition training) and nutritional interventions (polyphenols such as quercetin and curcumin) is felt.

\section{Ethical Considerations}

\section{Compliance with ethical guidelines}

This paper ethically approved by the research ethics committee of Tehran University of Medical Sciences (Code: IR.SUMS.REC.2017.S444).

\section{Funding}

The paper was extracted from the $\mathrm{PhD}$. dissertation of the first author, the Department of Sports Physiology, Faculty of Physical Education and Sport Sciences, Kish Campus, University of Tehran.

\section{Authors' contributions}

All authors contributed in preparing this article.

\section{Conflicts of interest}

The authors declared no conflicts of interest.

\section{Acknowledgements}

The authors would like to thank dr. Mohammad Reza Kordi and dr. Hamid Rajabi. 


\title{
اثر يك دوره بـتحركى اندام تحتانى بر بيان ثنهاي mir-1 و mir-206 عضله نعلى رتهاى تمرين كردهاستقامتى دوركى
}

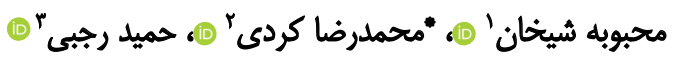 \\ ا. كروه فيزيولوزى ورزشي، دانشكده تربيت بلنى وعلوم ورزشى، دانشكاه تهران يرديس كيش، كيش، ايران.

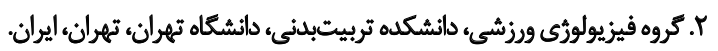

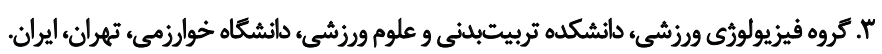

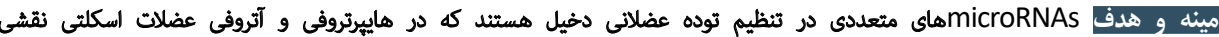

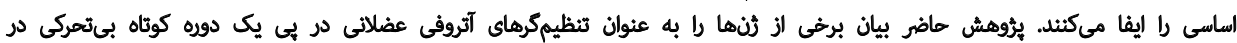

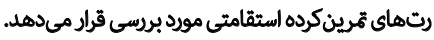

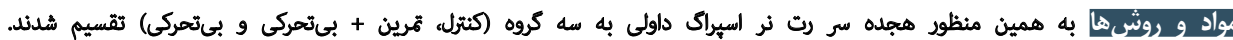

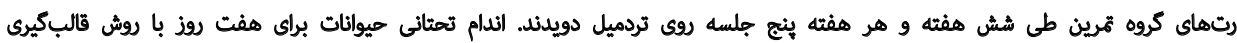

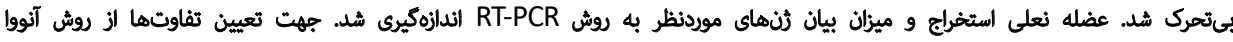

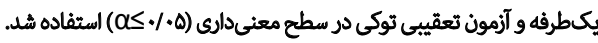

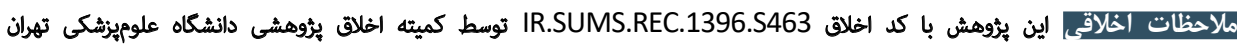
به بهويب رنسيد.

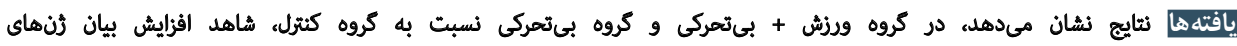

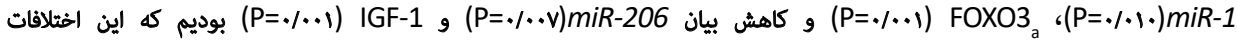

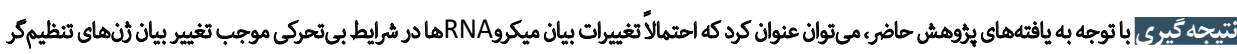

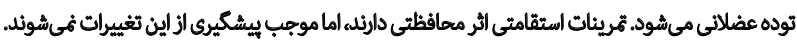

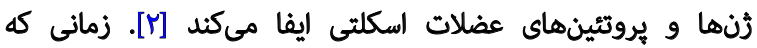

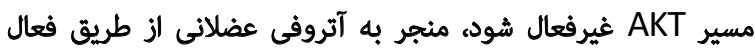

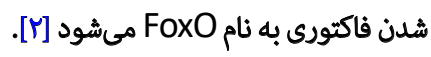

فعاليتهاى ورزشى از طريق تصريك ترشح هورمون رشده

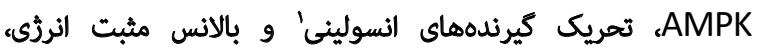

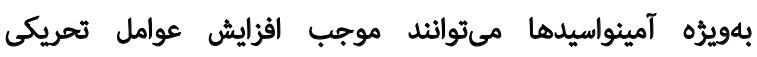

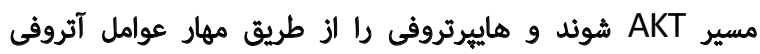

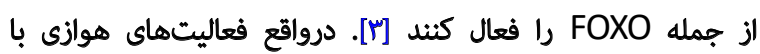
سازكارىهاى مطلوب سلولى موجب يك اثر مهافظتى در برابر

1. Insulin Receptors (IRS)

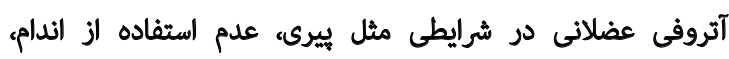

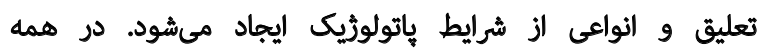

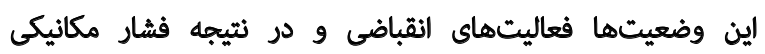

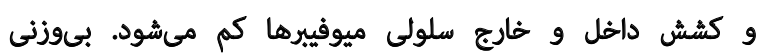

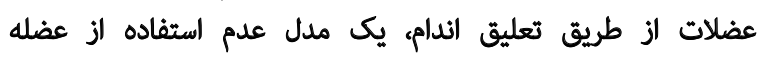
است كه باعث آتروفى قابل توجه عضله اسكلتى ميشود إنى [1].

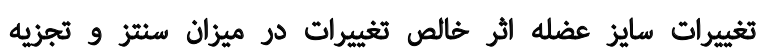

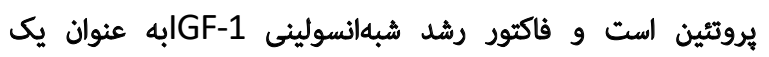

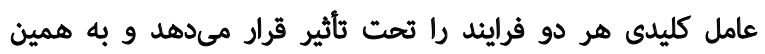

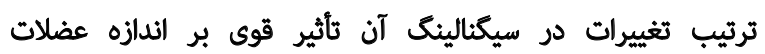

$$
\text { - }
$$

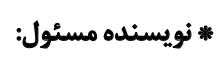

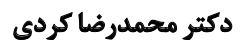

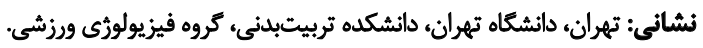

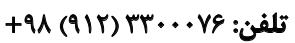
يست الكترونيكي: mrkordi@ut.ac.ir 
خاص، miR-1 و miR-206به دليل اينكه نقش مهمى در افزايش

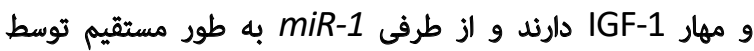

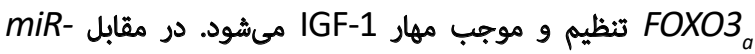
206 از طريق تخريب MyoD ميتواند و مهار فاكتور FOXO3 ميتواند موجب كاهش

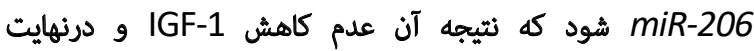

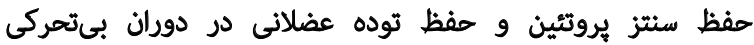
مىشود [1/]]. در مدل سايتوكاينى تجزيه عضلانى (TWEAK)

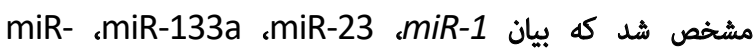

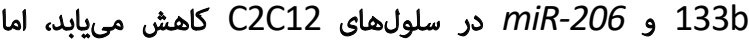
در عضلات اسكلتى موشها فقط بيان miR-133a miR-1 كاهش يافت [19miR-133b]

آلن و همكاران عنوان كردند يازده روز بىجادبكى موجب

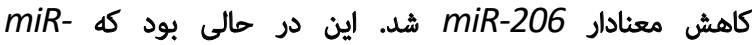

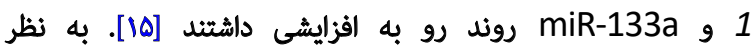

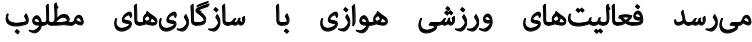
بلويره در افزايش عوامل رشدى و توسعه وضعيت هائيرتروفى دئى

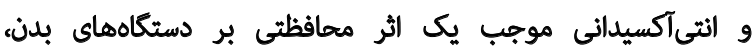

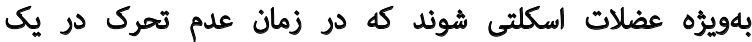

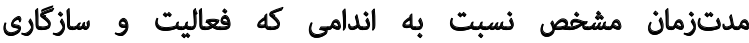

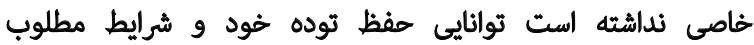

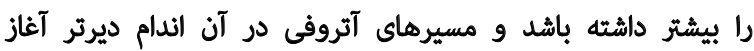

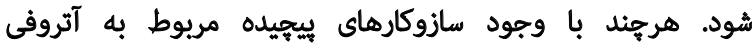

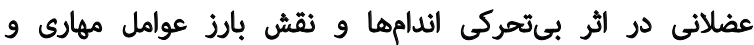

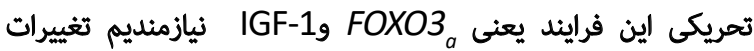

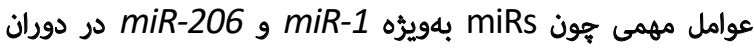

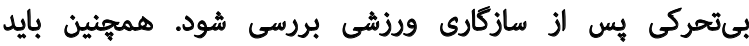

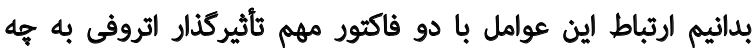

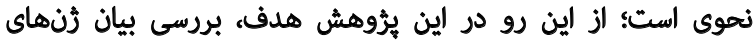

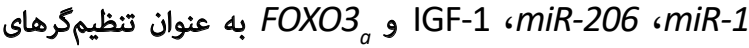

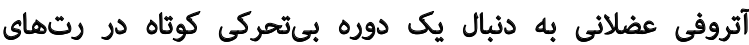

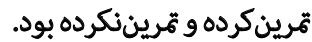

\section{مواد و روشها}

يُروهش حاضر از نوع بنيادى و به روش تجربى بود. تعداد هجده

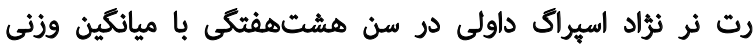

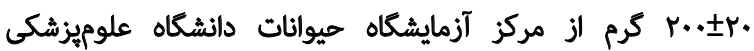

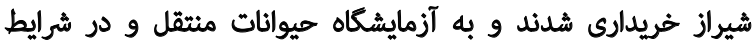

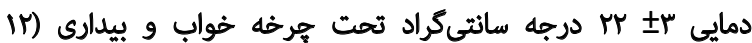

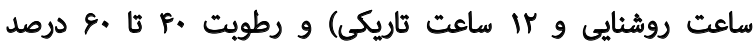

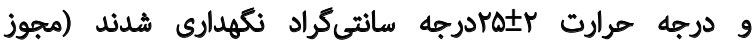
كد اخلاقى به شهاره IR.SUMS.REC.1396.S444).

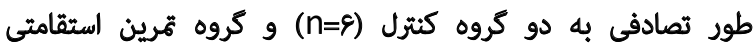
(n=IT)
آتروفى مىشود و از طرف ديكر مسيرهاى هاييروتروفى را توسعه

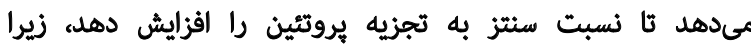

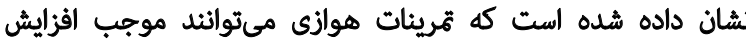

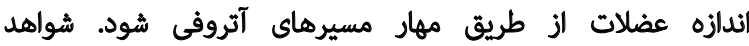

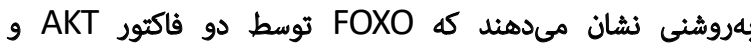

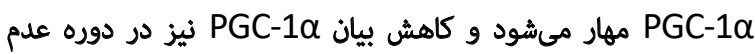

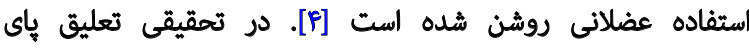
عقبى رتها (HLS) براى يك ثا دو دوان هفته باعث تغيير سطح بيان

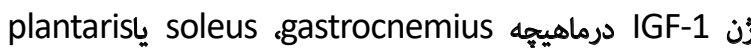

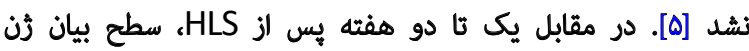

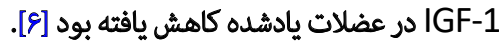

مطالعه ديكرى نشان داد HLS در جوندكان همراه با كاهش

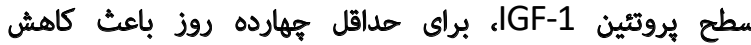

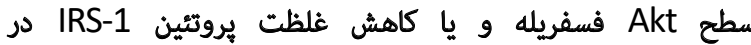

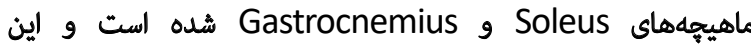

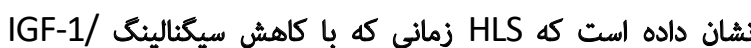

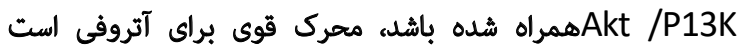

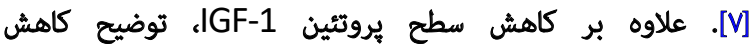
فسفوريلاسيون Akt و آتروفى عضلانى در هناون يلكام تعليق ميثواند

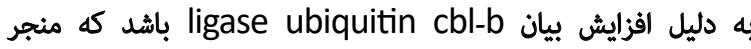

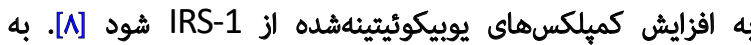

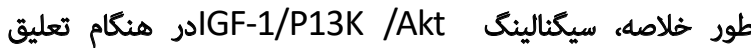

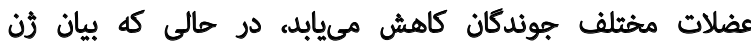

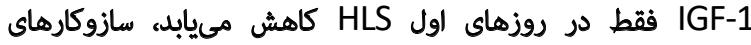
زياد و عوامل مثعددى در هرخه يروتئينها دخيل هستند [9]. يك فاكتور نسبتاً جديد از اين عوامل مثعدد (microR)

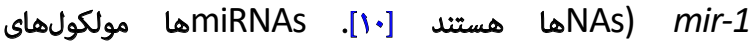

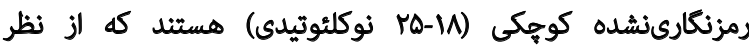

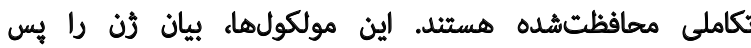

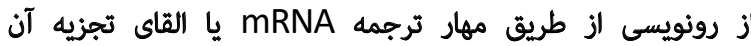
كنترل ميكنند و اين كار را از طريق التصال به به ناحيه ترجمهنشده

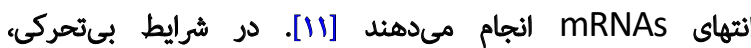
عضلات اسكلثى را مىتوان مهمترين بافتى دانست كه دستخوش

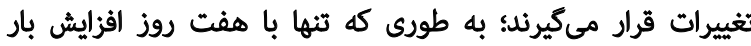
كارى بيان عضله 133a افزايش داشت، اما بيان miR-1 و و miR-133a كاهش

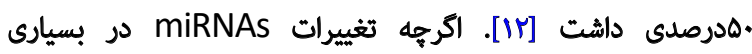

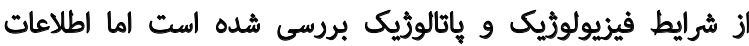

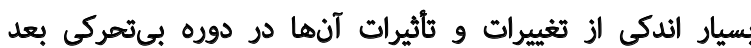

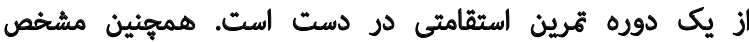

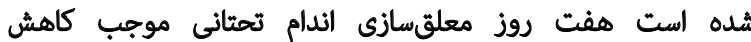

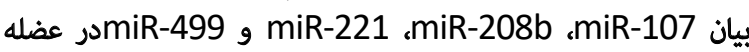

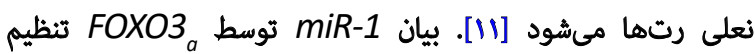

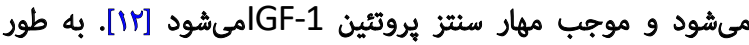


جدول ا. طرح يروتكل تمرين هوازي تداومى

\begin{tabular}{|c|c|c|c|c|c|c|c|c|}
\hline \multirow{2}{*}{ سرد كردن } & \multicolumn{6}{|c|}{ بدنه اصلى تمرين } & \multirow{2}{*}{ ه مر مقيقه كردن } & \multirow{2}{*}{ مراحل تمرين / مؤلفه تمرين } \\
\hline & 8. & $\Delta \cdot$ & p. & $r \cdot$ & r. & 10 & & \\
\hline & r. & $T V / \Delta$ & ra & $M / A$ & r. & IV/a & & سرعت (متر بر دقيقه) \\
\hline
\end{tabular}

sins

و بر اساس يروتكل سنتز CDNA موجود در كيث انجام شد. با

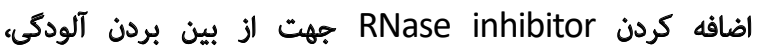

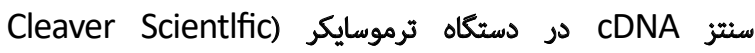

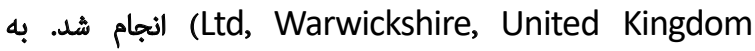
منظور اندازمكيرى سطح بيان رُنهاي مريوطه از روش RealQ Plus 2x با كمى آنزيم روشهاي (qRT-PCR) Real Time-PCR

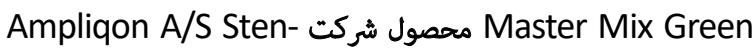

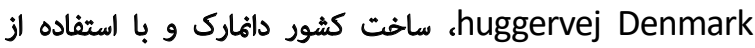
دستكاه applied Bio systems Step One آمريكا (Foster city celifornae) صورت كرفت. مخلوط

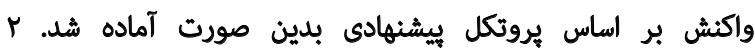

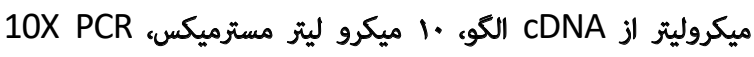

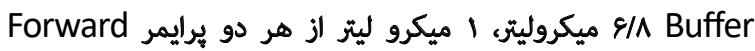

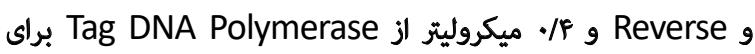
به دست آوردن بهترين دما آنالينك مورد الستفاده قرار كرفت و و آب كه هجم نهايى واكنش به ها ميكروليتر رسيد.

برنامه زمانى كرمايى دستكاه طبق اين مراحل انجام شد:

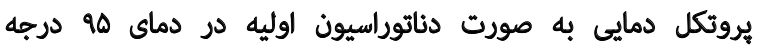

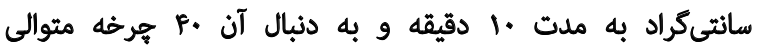

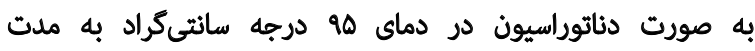

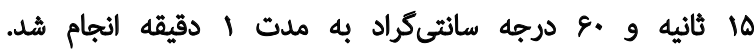

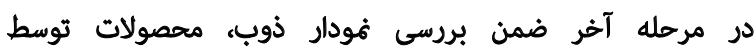

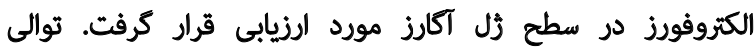

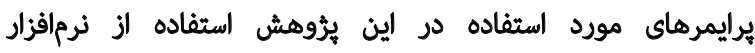

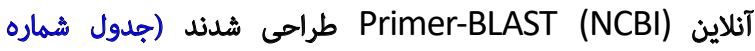

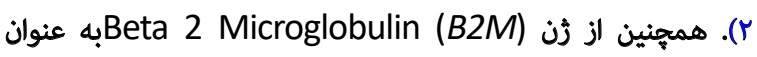
رن كنترل داخلى استفاده شد. تجزيه و تحليل دادها بها بر اساس

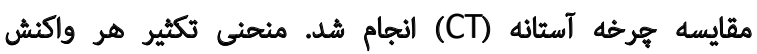
با منحنى تكثير زُن مرجع B2M PCR

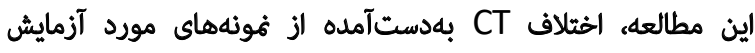
و غونههاى كنترل محاسبه و با الستفاده الز فرمول

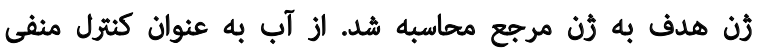

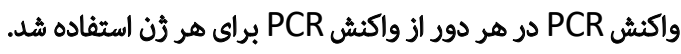

مخلوط واكنش بر الساس يروتكل بيشنهادى آماده شد كه

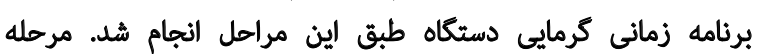

شُش هفته و ينج روز در هفته، تمرين استقامتى انجام دادند. ابتداء

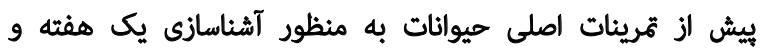

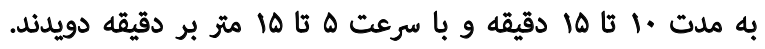

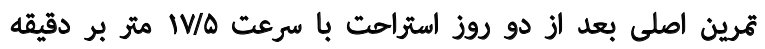

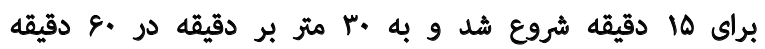

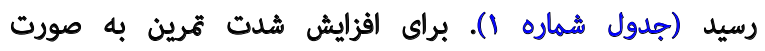

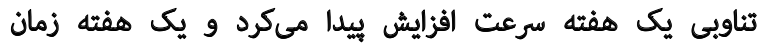

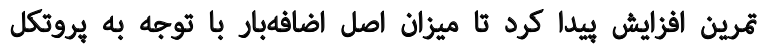

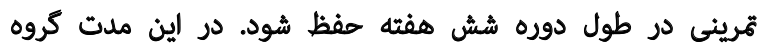

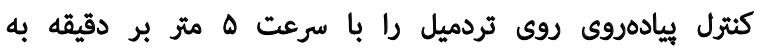

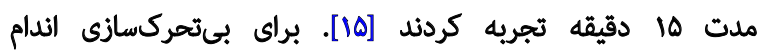

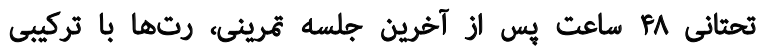

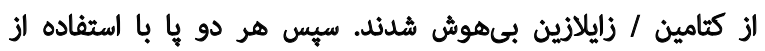

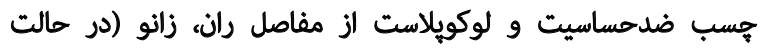

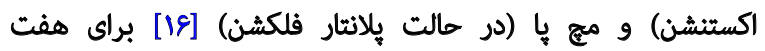
روز فيكس شدند. بىحركت شدن اندام تهتانى و توانايى مصرف

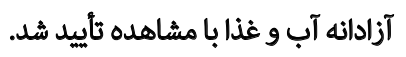

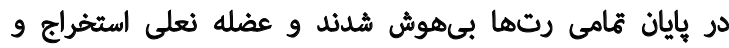

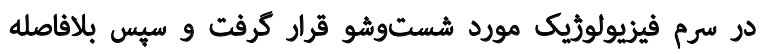

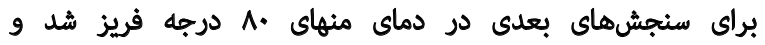

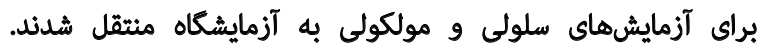

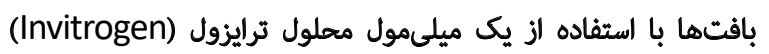

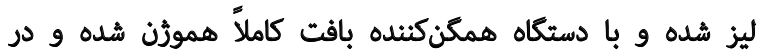

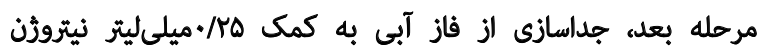

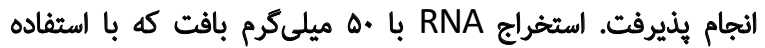

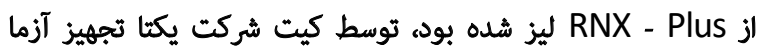

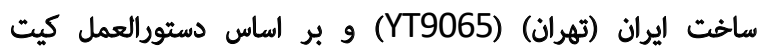

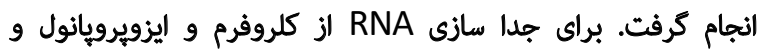

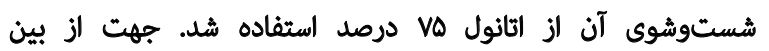

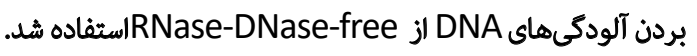
picodrop limited, Hinx-) كل نونها با دستكاه ييكودراب (ton, United Kingdom

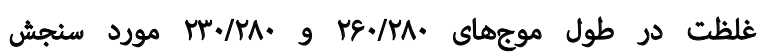

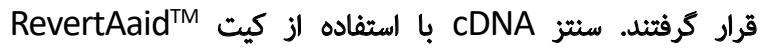
First Strand CDNA Synthesis K1622 به (Waltham, Massachusetts, USA) 
جدول r. يرايمرهاى Real-time PCR استفاده شده در مطالعه

Genes

Mir-1

Mir-206

IGF-1

$\mathrm{FOXO3}_{a}$

$B 2 m$

\section{Primer Sequences}

\section{Forward: 5' - ATGAAAAGGTTGAGACTTTTCA -3'}

Reverse: 5' - GCAAAGTGGCAGAACAATG -3'

Forward: $5^{\prime}$ - CCCAACAAGCTCTGCCTG $-3^{\prime}$

Reverse: 5'- GGGAGCATAGTTGACCTGAAAC -3'

Forward: $5^{\prime}$ - CTCTAACATCTCCCATCTCTC-3'

Reverse: 5' - TTCAAGAAGTCACATAGGCAG-3'

Forward: 5'- TTCGCAAGGACCCAATGA -3'

Reverse: $5^{\circ}$ - TCCAAGCTCCCATTGAACAT -3'

Forward: 5' - CGTGCTTGCCATTCAGAAA -3'

Reverse: 5'-ATATACATCGGTCTCGGTGG -3'

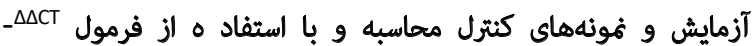

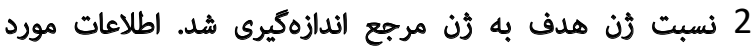
نياز يس از جمعآورى، توسط نرمافزال آمارى SPSS نسخه

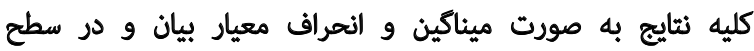

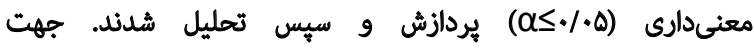

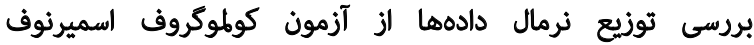
الستفاده شد. براي مقايسه تفاوت بين كروهها نيز آزيمون آنمان تحليل واريانس يكطرفه (آنووا) و آزمون تعقييى توكى مورد إنى الستفاده قرار كرفت.

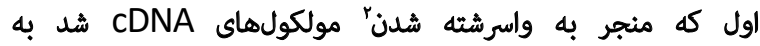

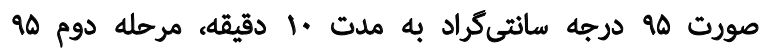

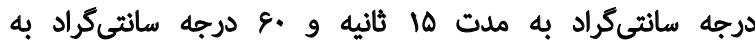

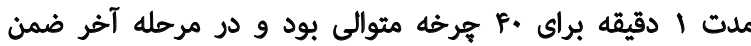

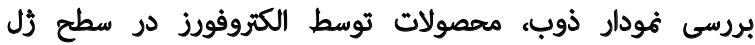

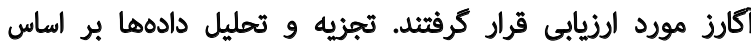

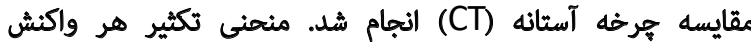

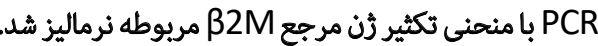
در اين مطالعه، اختلاف CT بلهستآمده از تمونهماى مورد

2. Denaturation

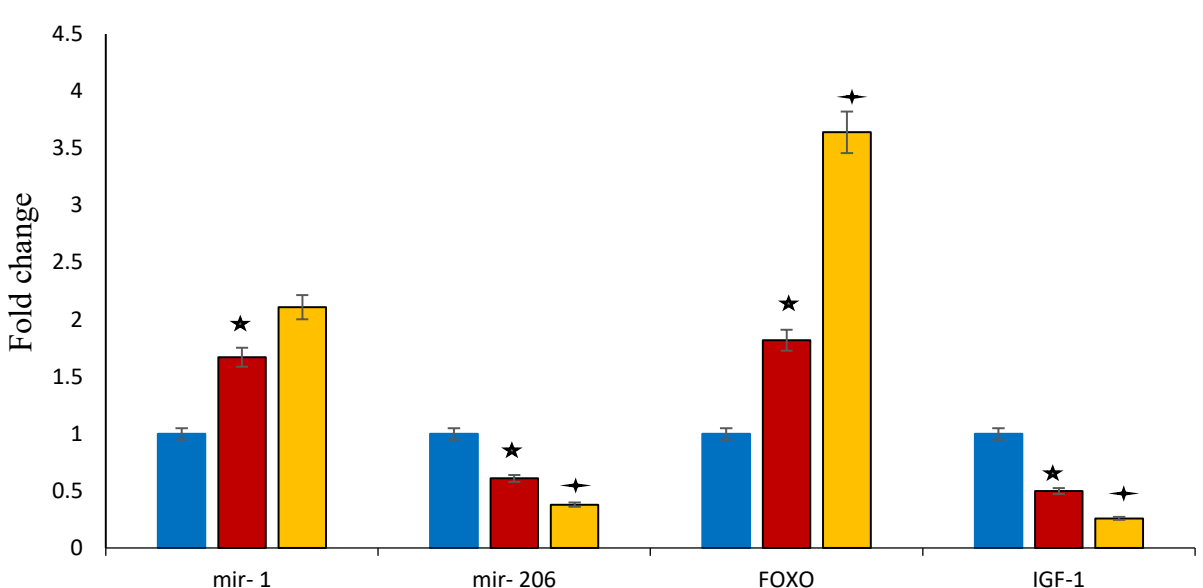

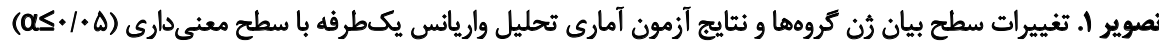

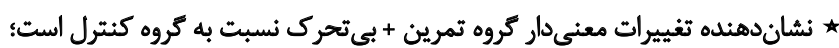
+ نشاندهنده تغييرات معنى دار كروه بى تحرك نسبت به كروه تمرين + بعتحرك است. 
جدول ஈ. ميانكين و انحراف استاندارد وزن و غذاي دريافتى كروهها و نتايج آزمون آمارى تحليل واريانس يكطرفه

\begin{tabular}{|c|c|c|c|c|}
\hline \multirow{2}{*}{ تحليل واريانس يكىطرفه } & \multicolumn{3}{|c|}{ ميانكين+|نحراف معيار } & \multirow{2}{*}{ شاخص } \\
\hline & بي تحرى & تمرين & كتترل & \\
\hline- & $M V / F \pm+1$ & $m e v / f \pm 1 f$ & reT/etlf & وزن بلن (كرم) \\
\hline $\begin{array}{l}F=30 / 614 \\
\text { Sig:0/002 }\end{array}$ & $\mid M Q / Y \pm I Y^{+}$ & $M \mathbb{M} / \mathrm{T} \pm q$ & $r r \cdot \mid f \pm 11$ & وزن عضله نعلى (ميلى كرم) \\
\hline $\begin{array}{l}F=162 / 050 \\
\text { Sig: } 0 / 016\end{array}$ & $\Delta++/ r \pm r \Delta^{+}$ & $\varepsilon q+/ \Lambda \pm r \Delta$ & grNA \pm MT & نسبت وزن عضله نعلى به وزن بلن (ميلى كرم / كيلوكرم) \\
\hline $\begin{array}{l}F=151 / 964 \\
\text { Sig: } 0 / 001\end{array}$ & $\| \varphi / P \pm \Delta^{\dagger}$ & $r \cdot / 8 \pm r$ & $\mathrm{r} / \Lambda \pm \mathrm{r}$ & غناي دريافتى (كرم / روزانه) \\
\hline
\end{tabular}

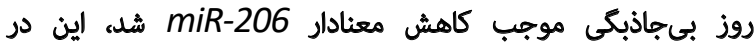

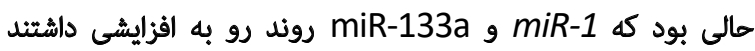

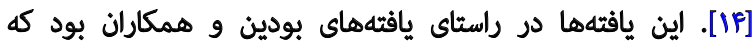

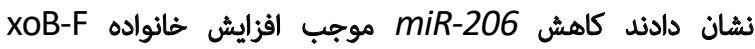
مىشود كه منجر به آتروفى مي اشوند

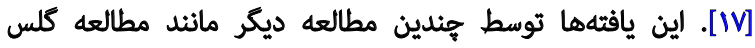

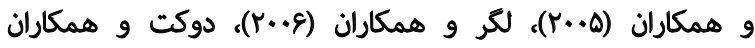
(Y.V) اينكه 206-miR به طور مستقيم يا غير مستقيم تأثير در مهار

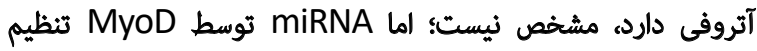

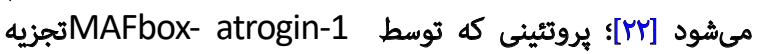

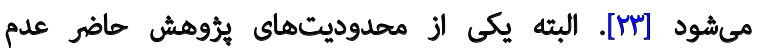

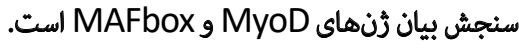

فعاليتهاي ورزشى از نوع الستقامتى با افزايش عملكرد هوازي

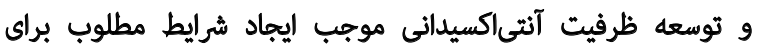

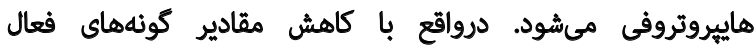

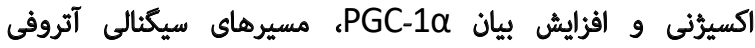

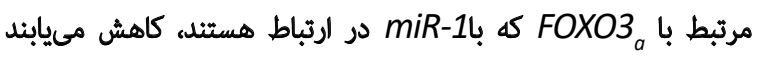

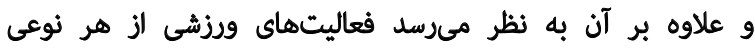

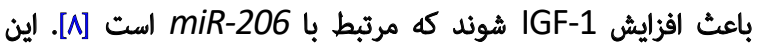

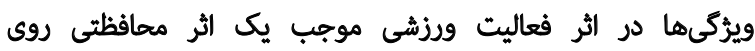

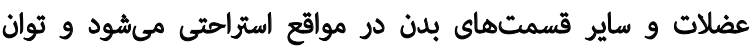

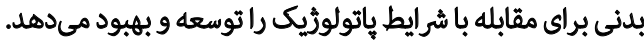

با ايجاد بيتحركى اولين اتفاقى كه در عضلات ميافتد كاهش

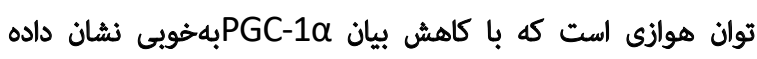

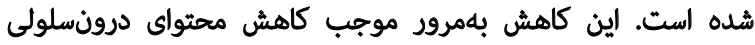

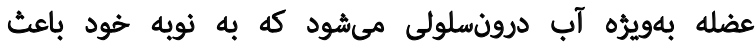

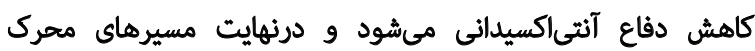

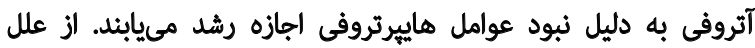

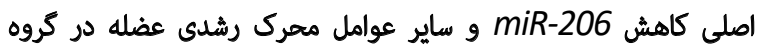

Latơl

وزن عضله نعلى، نسبت وزن عضله نعلى به وزن بلن و تغذيه

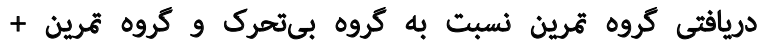

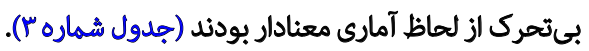
بيان نسبى ذُنهاي مورد بروسى در تصوير شماره ا الرائه شده

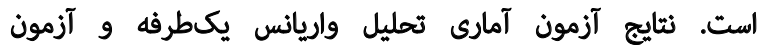

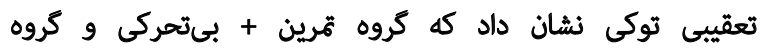

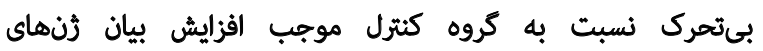

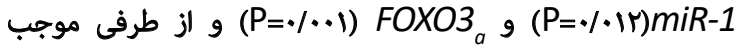

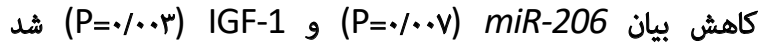
كه اين الختلافات از لحاظ آمارى معنادار بود. همحينين نثايج درونكروهى نشان دادن كه كروه بيتحرك نسبت به كروه تمرين

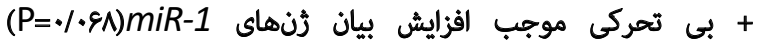
شله كه از لحاظ آمارى غير معنادار بود، اما افزايش

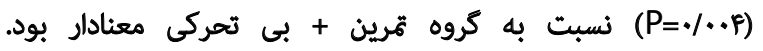

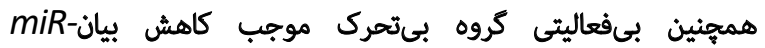

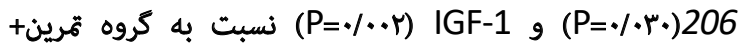
بى تحركى شد كه اين الختلاقات الز لحاظ آمارى معنادار بود.

ث

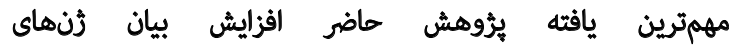
تحريككنده آتروفى (FOXO3

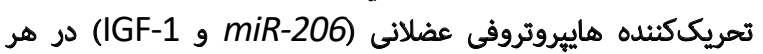

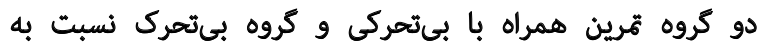

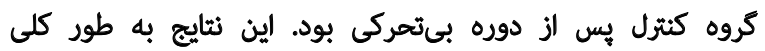

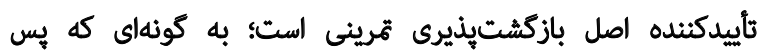

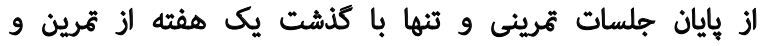

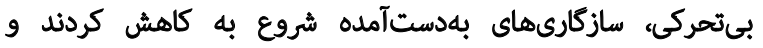

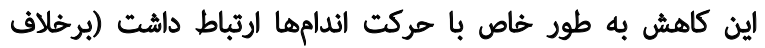

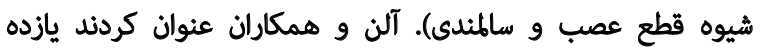




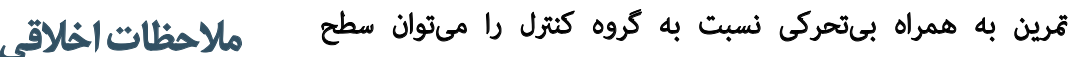

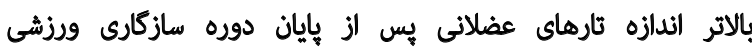

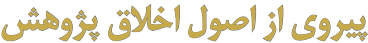

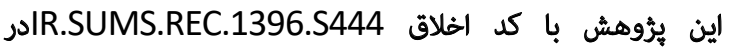
كميته اخلاق ايثووهشى دانشكاه علوميزشكى تهران به تصويب

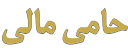

اين مقاله مستخرج از رساله دكترى نويسنده اوله دركروه

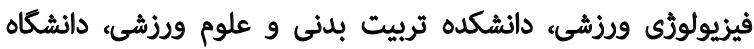

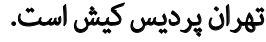

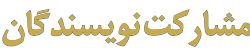

تمامى نويسندكان معيارهاى استاندارد نويسندكى بر اساس

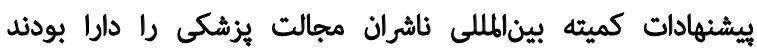
و همكى به يك اندازه در نكارش اثر سهم داشتند.

\section{تعاروض هنأقع}

نويسندكان تصريح مىكنند هيجكونه تضاد منافعى در يزوهش

ماضر وجود ندارد.

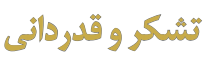

نويسندكان بر خود لازم مىدانند از استادان بزركوار، جناب

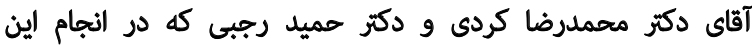
مطالعه يارىرسان بودند، تقدير كنيد.

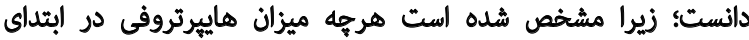

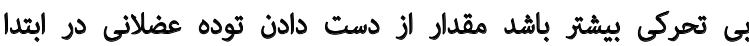

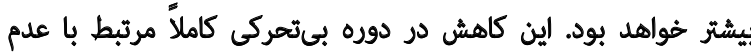

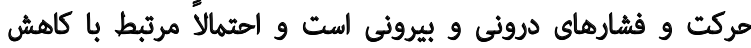

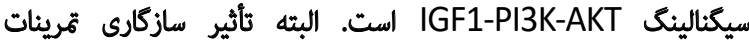

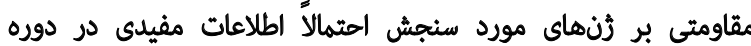

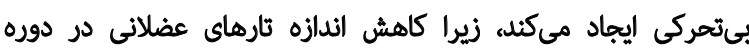

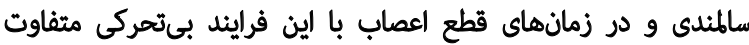

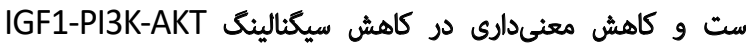

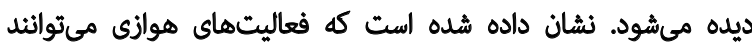

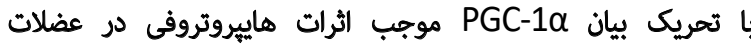

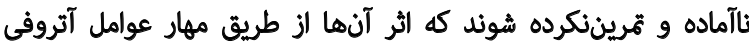

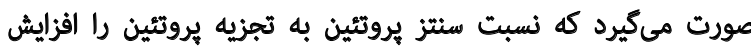

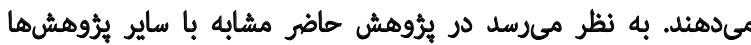

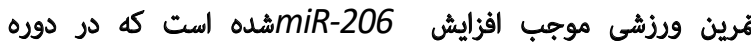

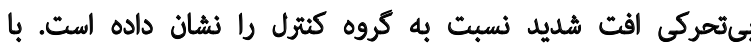

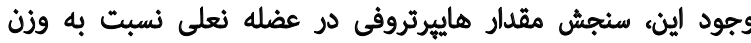

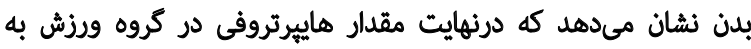

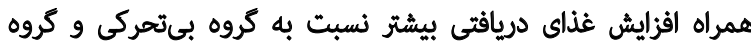

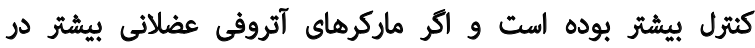

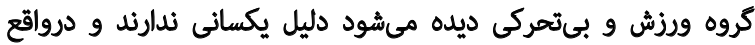

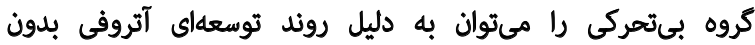

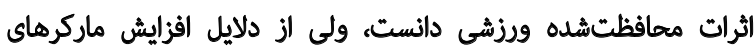

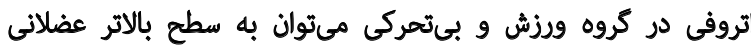
يس الز دوره ورزشي و أفت بيشتر دانست.

$$
\text { تئيجلكيرى }
$$

نتايج ئروهش حاضر نشان مىدهد با وجود اثرات محافظتكنندكى

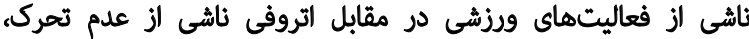

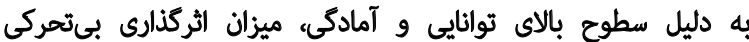

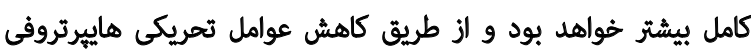

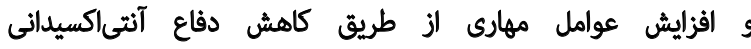

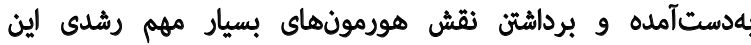

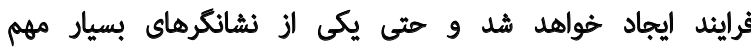

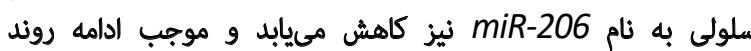

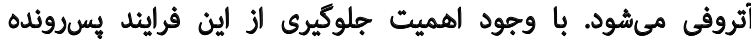

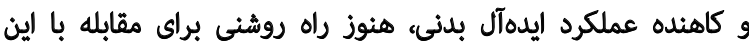

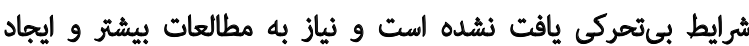

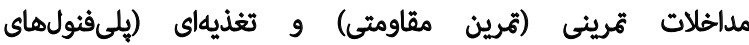
همجيون كوئرستين و كوركومين) است. 


\section{References}

[1] Lefebvre S, Bürglen L, Reboullet $S$, Clermont $O$, Burlet $P$, Viollet $L$, et al. Identification and characterization of a spinal muscular atrophydetermining gene. Cell. 1995; 80(1):155-65. [DOI:10.1016/00928674(95)90460-3]

[2] Glass DJ. Skeletal muscle hypertrophy and atrophy signaling pathways. Int J Biochem Cell Biol. 2005; 37(10):1974-84. [DOI:10.1016/j.biocel.2005.04.018] [PMID]

[3] Baar K, Wende AR, Jones TE, Marison M, Nolte LA, Chen M, et al. Adaptations of skeletal muscle to exercise: Rapid increase in the transcriptional coactivator PGC-1. FASEB J. 2002; 16(14):1879-86. [DOI:10.1096/ fj.02-0367com] [PMID]

[4] Sandri M, Lin J, Handschin C, Yang W, Arany ZP, Lecker SH, et al. PGC$1 \alpha$ protects skeletal muscle from atrophy by suppressing FoxO3 action and atrophy-specific gene transcription. Proc Natl Acad Sci U S A. 2006; 103(44):16260-5. [DOI:10.1073/pnas.0607795103] [PMID] [PMCID]

[5] Kyparos A, Feeback DL, Layne CS, Martinez DA, Clarke MSF. Mechanical stimulation of the plantar foot surface attenuates soleus muscle atrophy induced by hindlimb unloading in rats. J Appl Physiol. 2005; 99(2):739-46. [DOI:10.1152/japplphysiol.00771.2004] [PMID]

[6] Layne C. The Department of Health and Human Performance. [Phd. dissertation] Houston: College of Education-University of Houston; 2002-2003. https://uh.edu/class/hhp/ docs/annualreports/Annualreport03.pdf

[7] Fitts RH, Riley DR, Widrick JJ. Physiology of a microgravity environment invited review: Microgravity and skeletal muscle. J Appl Physiol. 2000; 89(2):823-39. [DOI:10.1152/jappl.2000.89.2.823] [PMID]

[8] Tintignac LA, Lagirand J, Batonnet S, Sirri V, Leibovitch MP, Leibovitch SA. Degradation of MyoD mediated by the SCF (MAFbx) ubiquitin ligase. J Biol Chem. 2005; 280(4):2847-56. [DOI:10.1074/jbc.M411346200] [PMID]

[9] Nakao R, Hirasaka K, Goto J, Ishidoh K, Yamada C, Ohno A, et al. Ubiquitin ligase $\mathrm{Cbl}-\mathrm{b}$ is a negative regulator for insulin-like growth factor 1 signaling during muscle atrophy caused by unloading. Mol Cell Biol. 2009; 29(17):4798-811. [DOI:10.1128/MCB.01347-08] [PMID] [PMCID]

[10] McCarthy JJ, Esser KA. MicroRNA-1 and microRNA-133a expression are decreased during skeletal muscle hypertrophy. J Appl Physiol. 2007; 102(1):306-13. [DOI:10.1152/japplphysiol.00932.2006] [PMID]

[11] McCarthy JJ, Esser KA, Peterson CA, Dupont-Versteegden EE. Evidence of MyomiR network regulation of $\beta$-myosin heavy chain gene expression during skeletal muscle atrophy. Physiol Genomics. 2009; 39(3):219-26. [DOI:10.1152/physiolgenomics.00042.2009] [PMID] [PMCID]

[12] Yang W, Chendrimada TP, Wang Q, Higuchi M, Seeburg PH, Shiekhat$\operatorname{tar} \mathrm{R}$, et al. Modulation of microRNA processing and expression through RNA editing by ADAR deaminases. Nat Struct Mol Biol. 2006; 13(1):13-21. [DOI:10.1038/nsmb1041] [PMID] [PMCID]

[13] Güller I, Russell AP. MicroRNAs in skeletal muscle: Their role and regulation in development, disease and function. J Physiol. 2010; 588(Pt 21):4075-87. [DOI:10.1113/jphysiol.2010.194175] [PMID] [PMCID]

[14] Panguluri SK, Bhatnagar S, Kumar A, McCarthy JJ, Srivastava AK, Cooper NG, et al. Genomic profiling of messenger RNAs and microRNAs reveals potential mechanisms of TWEAK-induced skeletal muscle wasting in mice. PloS One. 2010; 5(1):e8760. [DOI:10.1371/journal. pone.0008760] [PMID] [PMCID]
[15] Allen DL, Bandstra ER, Harrison BC, Thorng S, Stodieck LS, Kostenuik $\mathrm{PJ}$, et al. Effects of spaceflight on murine skeletal muscle gene expression. J Appl Physiol. 2009; 106(2):582-95. [DOI:10.1152/japplphysiol.90780.2008] [PMID] [PMCID]

[16] Kang C, Chung E, Diffee G, Ji LL. Exercise training attenuates agingassociated mitochondrial dysfunction in rat skeletal muscle: Role of PGC-1 $\alpha$. Exp Gerontol. 2013; 48(11):1343-50. [DOI:10.1016/j exger.2013.08.004] [PMID]

[17] Frimel TN, Kapadia F, Gaidosh GS, Li Y, Walter GA, Vandenborne K. A model of muscle atrophy using cast immobilization in mice. Muscle Nerve. 2005; 32(5):672-4. [DOI:10.1002/mus.20399] [PMID]

[18] Bodine SC, Latres E, Baumhueter S, Lai VK, Nunez L, Clarke BA, et al. Identification of ubiquitin ligases required for skeletal muscle atrophy. Science. 2001; 294(5547):1704-8. [DOI:10.1126/science.1065874] [PMID]

[19] Glass DJ. Skeletal muscle hypertrophy and atrophy signaling pathways. Int J Biochem Cell Biol. 2005; 37(10):1974-84. [DOI:10.1016/j. biocel.2005.04.018] [PMID]

[20] Léger B, Cartoni R, Praz M, Lamon S, Dériaz O, Crettenand A, et al. Akt signalling through GSK-3 3 , mTOR and Foxo1 is involved in human skeletal muscle hypertrophy and atrophy. J Physiol. 2006; 576(Pt 3):923-33. [DOI:10.1113/jphysiol.2006.116715] [PMID] [PMCID]

[21] Doucet $M$, Russell AP, Léger B, Debigaré R, Joanisse DR, Caron M-A, et al. Muscle atrophy and hypertrophy signaling in patients with chronic obstructive pulmonary disease. Am J Respir Crit Care Med. 2007 176(3):261-9. [DOI:10.1164/rccm.200605-704OC] [PMID]

[22] Léger B, Derave W, De Bock K, Hespel P, Russell AP. Human sarcopenia reveals an increase in SOCS- 3 and myostatin and a reduced efficiency of Akt phosphorylation. Rejuvenation Res. 2008; 11(1):163-175B. [DOI:10.1089/rej.2007.0588] [PMID]

[23] Chen JF, Mandel EM, Thomson JM, Wu Q, Callis TE, Hammond SM, et al. The role of microRNA-1 and microRNA-133 in skeletal muscle proliferation and differentiation. Nat Genet. 2006; 38(2):228-33. [DOI:10.1038/ng1725] [PMID] [PMCID] 